ARTICLE

https://doi.org/10.1038/s41467-019-09830-6

\title{
Hydrophobic carbon dots with blue dispersed emission and red aggregation-induced emission
}

\author{
Haiyao Yang (1) 1,2, Yingliang Liu', Zhouyi Guo², Bingfu Lei (1) ${ }^{1}$, Jianle Zhuang ${ }^{1}$, Xuejie Zhang ${ }^{1}$, Zhiming Liu ${ }^{2} \&$ \\ Chaofan $\mathrm{Hu}^{1}$
}

Carbon dots (CDs) have been studied for years as one of the most promising fluorescent nanomaterials. However, CDs with red or solid-state fluorescence are rarely reported. Herein, through a one-pot solvothermal treatment, hydrophobic CDs (H-CDs) with blue dispersed emission and red aggregation-induced emission are obtained. When water is introduced, the hydrophobic interaction leads to aggregation of the $\mathrm{H}-\mathrm{CDs}$. The formation of $\mathrm{H}-\mathrm{CD}$ clusters induces the turning off of the blue emission, as the carbonized cores suffer from $\pi-\pi$ stacking interactions, and the turning on of the red fluorescence, due to restriction of the surfaces' intramolecular rotation around disulfide bonds, which conforms to the aggregation-inducedemission phenomenon. This on-off fluorescence of the $\mathrm{H}-\mathrm{CD}$ s is reversible when the $\mathrm{H}-\mathrm{CD}$ powder is completely dissolved. Moreover, the H-CD solution dispersed in filter paper is nearly colorless. Finally, we develop a reversible two switch-mode luminescence ink for advanced anti-counterfeiting and dual-encryption.

\footnotetext{
${ }^{1}$ Guangdong Provincial Engineering Technology Research Center for Optical Agriculture, College of Materials and Energy, South China Agricultural University, 510642 Guangzhou, China. ${ }^{2}$ MOE Key Laboratory of Laser Life Science \& SATCM Third Grade Laboratory of Chinese Medicine and Photonics Technology, College of Biophotonics, South China Normal University, 510631 Guangzhou, China. Correspondence and requests for materials should be addressed to Y.L. (email: tliuyl@scau.edu.cn) or to Z.L. (email: liuzm@scnu.edu.cn) or to C.H. (email: thucf@scau.edu.cn)
} 
$\mathrm{M}$ any types of carbon dots (CDs) have been reported because they are more eco-friendly and potentially a carbon-based fluorescent nanomaterial; however, in solution, most CDs show emission in the blue to green-light regions only ${ }^{1-3}$. Therefore, there is an urgency to attain longwavelength and multicolor emission of CDs for further applications, particularly in biologically relevant and anticounterfeiting fields ${ }^{1,2,4-9}$. The most common method for inducing longwavelength and multicolor emission is doping heteroatoms into the lattices of carbon. The addition of heteroatoms leads to defects with the framework of the CDs. Therefore, contraction of the CDs' band gaps further induces a redshift of the CDs' fluorescence. Recently, Liu and coworkers fabricated red emission B, N, S-codoped CDs using 2,5-Diaminobenzenesulfonic acid and 4-aminophenylboronic acid hydrochloride. Earlier, Ge et al. ${ }^{9}$ designed a series of Suzuki reactions to synthesize polythiophene derivatives as precursors for red emission S-doped CDs.

However, previous studies on red emission CDs did not obtain CDs that exhibit red fluorescence in the solid state. Most reported CDs only fluoresce when dissolved in solution. The currently accepted mechanism of this phenomenon is similar to the $\mathrm{H}$ aggregation of organic fluorescent molecules; CDs suffer from $\pi-\pi$ stacking in the solid state and the aggregation of large conjugated systems consumes the transition energy, therefore, resulting in the quenching of the CDs' fluorescence. While the extinction of the CDs' luminescent solid-state has hindered their application in LED and anticounterfeiting technology $y^{8,10-13}$. The current method to maintain CDs' solid-state-fluorescence (SSF) is to block the $\mathrm{CD}$ monomers from direct contact. Most reported studies on SSF CDs attempt to dope CDs into matrices or introduce polymer chains into $\mathrm{CDs}^{8,11}$. For instance, Chen et al. ${ }^{13}$ prepared $\mathrm{N}$-doped CD's (NCDs) with yellow-green SSF by hydrothermal treatment of poly (vinyl alcohol) (PVA) and ethylenediamine (EDA). The abundant surface PVA chains covered around the NCDs prevented the graphitizing cores from $\pi-\pi$ interactions; thus, resisting the aggregation-caused-quenching (ACQ) of the NCDs' fluorescence. However, these studies did not achieve red SSF of CDs. Moreover, the introduction of matrices or polymer chains restricted the concentration of the doped CDs; if too many CDs are introduced, their ACQ will still take place.

Unlike the $\mathrm{ACQ}^{14}$ property of nanomaterials, B.Z Tang ${ }^{15-18}$ and coworkers discovered a series of organic fluorescent materials and found that luminogen aggregation played a destructive role in the light-emitting process. In their studies, a series of symmetrical molecules were found to be nonluminescent in the dissolved state, but emissive in the aggregated state. The term "aggregationinduced emission" (AIE) was coined for this phenomenon, as the nonluminescent symmetrical molecules were induced to emit via aggregate formation. This theory has not been utilized in the SSF of carbon dots yet.

Incidentally, we find another approach for maintaining a CDs' SSF, in addition to introducing them into solid dispersed systems: crowning the CDs' graphitized cores with rotatable symmetric surfaces, through a series of amidation and rearrangement during a solvothermal carbonization process. When fully dispersed as a homogeneous solution, our CDs exhibit similar PL characteristics as the reported blue emission CDs. By adding water, the CDs continuously assemble due to their hydrophobicity, the blue fluorescence turns off while a red SSF turns on. Like AIE molecules in solution, the surficial groups of CDs can rotate around the intramolecular disulfide bonds and consume the absorbed energy, thus, not producing fluorescence. However, in the solid state, as a result of the intramolecular rotation being banned, the excitation energy can transfer dominantly into fluorescence. Therefore, we have designed a method to synthesize hydrophobic $\mathrm{N}$, S-doped CDs (H-CDs) with a two-switch-mode luminescence between a blue dissolved fluorescence and a red AIE. Moreover, the output of the $\mathrm{H}-\mathrm{CD}$ powders is higher (after purification, the mass ratio of $\mathrm{H}-\mathrm{CD}$ powders to raw materials is approximately $80 \%$ ) than prior methods. Several characterizations are taken to determine the properties of $\mathrm{H}-\mathrm{CD}$. When $\mathrm{H}-\mathrm{CD}$ powder dissolves into certain organic solvents (ethanol or acetic acid), it displays the same blue fluorescence as the as-prepared $\mathrm{H}-\mathrm{CD}$ solution. However, in DMF the $\mathrm{H}-\mathrm{CD}$ displays both blue and red fluorescence, due to the existence of mono-dispersed and aggregated $\mathrm{H}$-CDs. To confirm the fluorescence mechanism and the relationship between the $\mathrm{H}-\mathrm{CD}$ dispersed state and fluorescence, we have designed a control experiment (replaced dithiosalicylic acid with benzoate to remove the disulfide bonds). Finally, we fill the as-prepared H-CD solution into a mark pen and conduct a series of anticounterfeit and encryption experiments to develop a reversible two-switch-mode luminescence ink.

\section{Results}

Preparation and characterization of the H-CDs. The H-CD powder was easily prepared through a one-pot solvothermal process of melamine (MA) and a dithiosalicylic acid (DTSA)/ acetic acid solution, followed by a simple purification (Fig. 1). It should be noted that acetic acid plays a vital role during the formation of H-CDs. In addition to being an environmentally friendly solvent with low cost, it is also a catalyst for $\mathrm{H}-\mathrm{CDs}$ carbonization and the constitution of $\mathrm{H}-\mathrm{CD}$ surface (Supplementary Fig. 1a). To further investigate the effect of acetic acid, we applied a series of control experiments which replaced acetic acid with formic acid, propionic acid and saturated aqueous solution of oxalic acid. When propionic acid is added, the product (named as PA-CDs) shows a similar PL property as the H-CDs: blue emission in dispersion and yellow AIE in the powder state (Supplementary Fig. 1b, c). However, propionic acid is much more expensive and toxic than acetic acid, and the fluorescence of PA-CD powder is yellow unlike the red AIE of the H-CDs. Through continuous water addition, the transparent as-prepared $\mathrm{H}-\mathrm{CD}$ solution gradually turns into a turbid liquid, and the blue emission fades away. Then, a red fluorescence emerges. H-CD powder displaying red SSF under $365 \mathrm{~nm}$ UV irradiation can be obtained with further purification and drying. Remarkably, under $2 \mathrm{~nm} \mathrm{UV}$, the red emission of the $\mathrm{H}-\mathrm{CD}$ powder remains while the $\mathrm{H}-\mathrm{CD}$ dispersion displays nearly no fluorescence.

The as-prepared H-CDs have been characterized with transmission electron microscopy (TEM), X-ray diffraction (XRD) and Raman spectroscopy to confirm the nature of the carbon nanoparticles. As shown in Fig. 2a, b, the TEM image of the $\mathrm{H}-\mathrm{CDs}$ presents size distributions between 4 and $10 \mathrm{~nm}$, with an average diameter of approximately $6.5 \mathrm{~nm}$. High-resolution TEM (HR-TEM) shows a lattice spacing of $0.21 \mathrm{~nm}$ corresponding to the (100) facet of graphite and reveals that the H-CDs contain graphite-like structures ${ }^{19-21}$. The XRD pattern of the $\mathrm{H}$ CDs (Fig. 2c) has an apparent peak at approximately $25^{\circ}$, which is attributed to an interlayer spacing of $0.34 \mathrm{~nm}$, while the peak near $41^{\circ}$ represents the $0.21 \mathrm{~nm}$ interlayer spacing ${ }^{5,8,13,22}$. The Raman spectrum in Fig. 2d displays two peaks at $1348 \mathrm{~cm}^{-1}$ (D band) and $1584 \mathrm{~cm}^{-1}$ ( $\mathrm{G}$ band), referring to areas of disordered surfaces and $s p^{2}$ carbon networks in the H-CDs' frameworks, respectively. The calculated intensity ratio $I_{\mathrm{D}} / I_{\mathrm{G}}$ is 5.61 , indicating the amorphous surface of the $\mathrm{H}-\mathrm{CDs}^{1,5,6,19}$.

Fourier transform infrared (FT-IR) spectra, X-ray photoelectron spectra (XPS) and nuclear magnetic resonance (NMR) spectroscopy were taken to further analyze the chemical structure of the H-CDs. The FT-IR spectrum (Fig. 2i) uncovers that the surface of the H-CDs contains methylene (2876 and $\left.2973 \mathrm{~cm}^{-1}\right), \mathrm{C} \equiv \mathrm{N}$ $\left(2034 \mathrm{~cm}^{-1}\right), \mathrm{S}-\mathrm{H}\left(2650 \mathrm{~cm}^{-1}\right)$, amide carbonyl $\left(1682 \mathrm{~cm}^{-1}\right)$, 
a

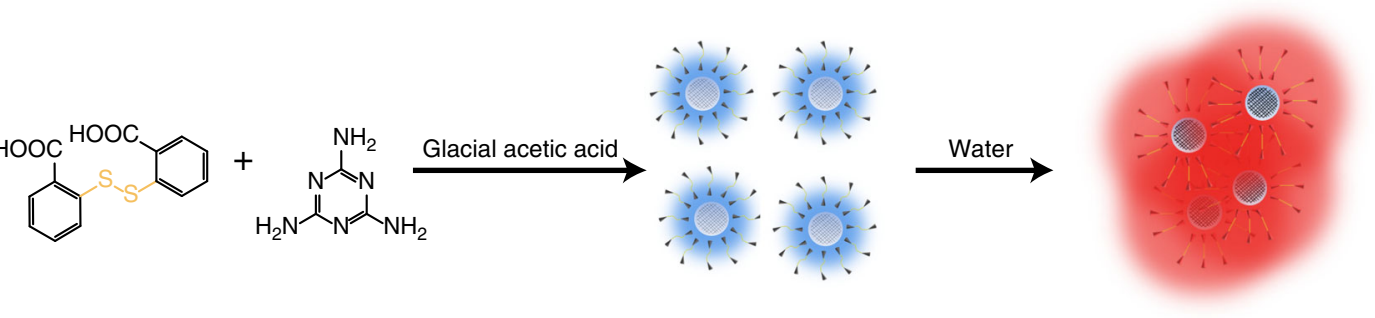

b Sunlight365 nm UV
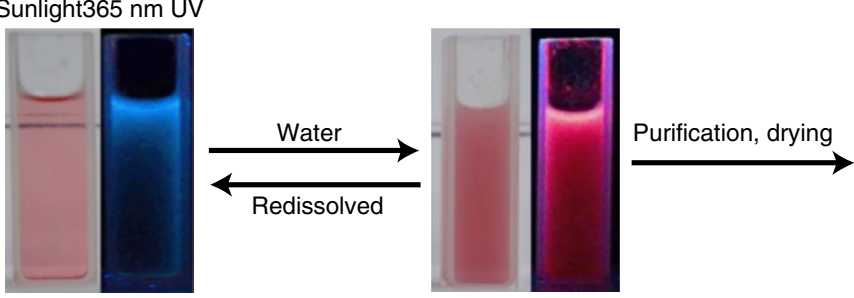

Sunlight

$365 \mathrm{~nm}$ UV
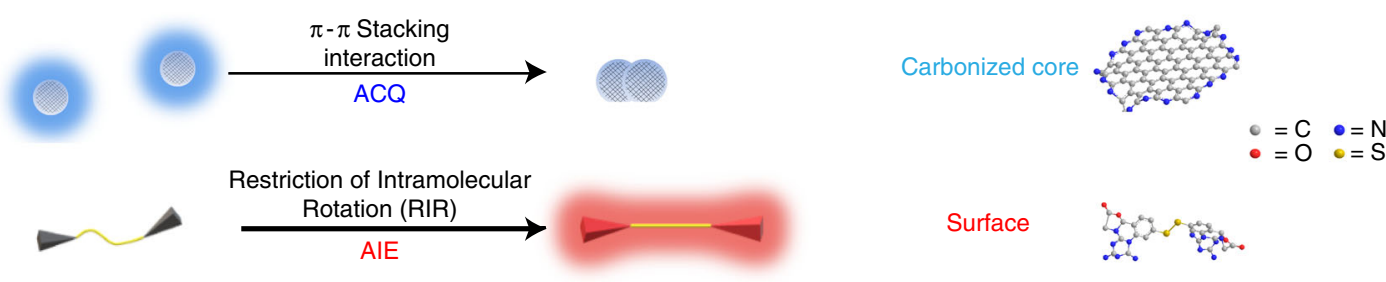

Surface

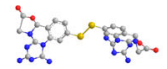

Fig. $1 \mathrm{H}-\mathrm{CDs}$ ' formation, structure, fluorescence and appearance in different state. a Formation of $\mathrm{H}-\mathrm{CD}$ monomers and their aggregates (the disulfide bond in dithiosalicylic acid molecular is highlighted with yellow). b Photographs of the $\mathrm{H}$-CDs' two-switch-mode luminescence principle. $\mathbf{c}$ Fluorescence principle and proposed structure of H-CD's core and surface (the colors of glowing edges represent the color of their fluorescence). $\mathrm{H}-\mathrm{CD}$, hydrophobic carbon dot

$\mathrm{C}=\mathrm{C}\left(1469 \mathrm{~cm}^{-1}\right), \mathrm{C}-\mathrm{N}\left(1407 \mathrm{~cm}^{-1}\right), \mathrm{C}-\mathrm{S}\left(685 \mathrm{~cm}^{-1}\right), \mathrm{S}-\mathrm{S}$ $\left(491 \mathrm{~cm}^{-1}\right)$, aromatic $\mathrm{C}-\mathrm{NH}\left(1261 \mathrm{~cm}^{-1}\right)$ and $\mathrm{C}-\mathrm{O}\left(1124 \mathrm{~cm}^{-1}\right)$ functional groups or chemical bonds. Additionally, the FT-IR spectra of MA and DTSA exhibit that these raw materials contain an hydroxyl or amino (3064 and $\left.3411 \mathrm{~cm}^{-1}\right)^{1,4,8}$. Furthermore, after the amidation and carbonization, these hydrophilic groups almost disappear in the H-CDs, thus, contributing to the hydrophobic properties of the H-CDs ${ }^{23-34}$. The full XPS spectrum presented in Fig. 2e shows four peaks at 284.81, 399.62, 532.22, and $163.89 \mathrm{eV}$, suggesting that the $\mathrm{H}-\mathrm{CD}$ s consisted of $\mathrm{C}, \mathrm{N}, \mathrm{O}$, and $\mathrm{S}$ elements, and the atomic ratios were calculated to $79.28 \%, 6.47 \%$, $10.99 \%$, and $3.26 \%$, respectively. In Fig. 2 f, the high-resolution XPS spectrum of the C $1 \mathrm{~s}$ band was separated into three peaks at $284.81,286.41$, and $288.95 \mathrm{eV}$, which are assigned to $\mathrm{C}-\mathrm{C} / \mathrm{C}=\mathrm{C}$, $\mathrm{C}-\mathrm{N}$ and $\mathrm{C}=\mathrm{O} / \mathrm{C}=\mathrm{N}$, respectively. The $\mathrm{N} 1 \mathrm{~s}$ band (Fig. $2 \mathrm{~g}$ ) exhibits two peaks at 399.07 and $400.27 \mathrm{eV}$, respectively, which correspond to pyridinic $\mathrm{C}_{3}-\mathrm{N}$ and pyrrolic $\mathrm{C}_{2}-\mathrm{N}-\mathrm{H}$ groups. The $\mathrm{S}$ $2 p$ band in Fig. $2 \mathrm{~h}$ contains three peaks at $163.35 \mathrm{eV}$ for $\mathrm{S}-\mathrm{C}$, $163.81 \mathrm{eV}$ for $\mathrm{S}-\mathrm{H}$ and $164.57 \mathrm{eV}$ for $\mathrm{S}-\mathrm{S}$. These three highresolution spectra collectively indicate the successful insertion of $\mathrm{S}$ and $\mathrm{N}$ atoms into the $\mathrm{H}$-CDs. Furthermore, NMR spectra $\left({ }^{1} \mathrm{H}\right.$ and ${ }^{13} \mathrm{C}$ ) were employed to distinguish the $s p^{3}$-hybridized carbon atoms from the $s p^{2}$-hybridized carbon atoms (Fig. $2 \mathrm{j}, \mathrm{k}$ ). Deuterium-labeled DMSO-d6 $\left(\mathrm{CD}_{3} \mathrm{SOCD}_{3}\right)$ was used as a solvent. In the ${ }^{1} \mathrm{H}$ NMR spectra, $s p^{2}$ carbons were detected. The peak at $9.99 \mathrm{ppm}$ in Fig. $2 \mathrm{j}$ is the chemical shift of the carboxyl protons. Furthermore, signals from the aromatic rings are detected at $8.3 \mathrm{ppm}$, which can be attributed to graphitized cores' proton resonances. The emergence of the $-\mathrm{NH}_{2}$ protons at $5.75 \mathrm{ppm}$ implies the introduction of primary amines into the heterocyclic surface ${ }^{19,22,35}$. In the ${ }^{13} \mathrm{C}$ NMR spectrum, signals in the range of $30-45 \mathrm{ppm}$ are associated with the aliphatic $\left(s p^{3}\right)$ carbon atoms, and signals from 100 to $185 \mathrm{ppm}$ are indicative of $s p^{2}$ carbon atoms. Signals in the range of $170-185 \mathrm{ppm}$ correspond to carboxyl/amide groups ${ }^{36-38}$. Based on the aforementioned characterizations, which support the reaction mechanism proposed in Supplementary Fig. 1a, a molecular model for the H-CDs can be constructed: a nanoscale graphite-like skeleton with defects caused by pyridinic nitrogen atoms and disulfide bonds, covered with $\mathrm{C}$, $\mathrm{N}, \mathrm{O}$ and $\mathrm{S}$ containing symmetrical heterocycle rotatable structures. Notably, there are few amino and hydroxyl functional groups on the surface of the $\mathrm{H}-\mathrm{CDs}$, which is quite different from the water-soluble CDs reported in prior works. This model explains the hydrophobicity and optical properties.

Optical properties and fluorescence mechanism of the H-CDs. The UV-Vis absorption, PL excitation, and emission of the asprepared H-CD solution and powder were examined to evaluate their optical properties. As shown in Fig. $3 a$, the UV-Vis absorption of the as-prepared $\mathrm{H}$-CDs has two peaks at $\lambda_{\max 1} \approx$ $280 \mathrm{~nm}$ and $\lambda_{\max 2} \approx 360 \mathrm{~nm}$ due to the $\pi-\pi^{\star}$ transitions of the $\mathrm{C}=\mathrm{C}$ in the core of the H-CD. While the H-CD powder exhibits a different broad absorption, with a dominating band at $\lambda_{\max } \approx$ $560 \mathrm{~nm}$ (Fig. 3b), which is attributed to the $\mathrm{n}-\pi^{*}$ transitions of the surface states containing $\mathrm{C}=\mathrm{N} / \mathrm{C}=\mathrm{O}, \mathrm{C}-\mathrm{O}$ and $\mathrm{C}-\mathrm{S}$ structures. Figure $3 \mathrm{~d}$ represents the PL emission of the $\mathrm{H}-\mathrm{CD}$ powder under different excitation wavelengths, showing a stable red emission at $\lambda_{\text {max }} \approx 620 \mathrm{~nm}$, with a different excitation wavelength that is more similar to traditional inorganic phosphors than reported CDs. However, the as-prepared H-CD solution (Fig. 3c) exhibits excitation-dependent PL features; similar to most CDs in prior works, the optimal excitation and emission are near 360 and $467 \mathrm{~nm}^{1,6,10,11,20,39}$, respectively. The computational process of the H-CDs' molecular orbital energy level and fluorescence lifetime ( $4.56 \mathrm{~ns})$ is described in the "Methods" section. The quantum yield of the H-CDs can be calculated as $5.96 \%$, 
a
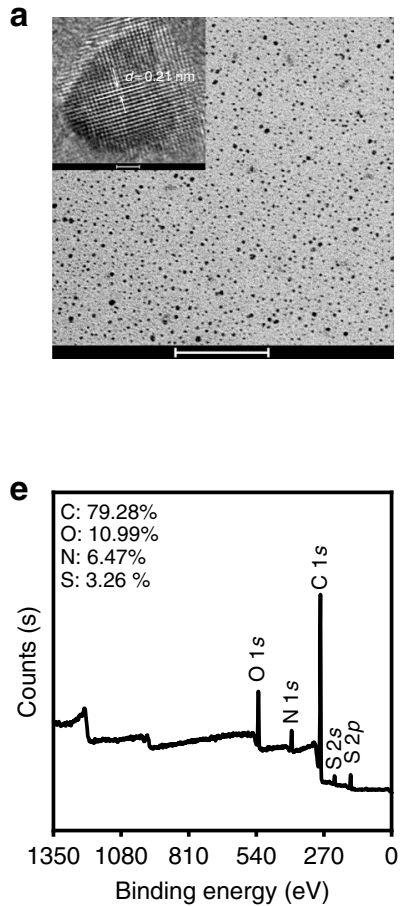

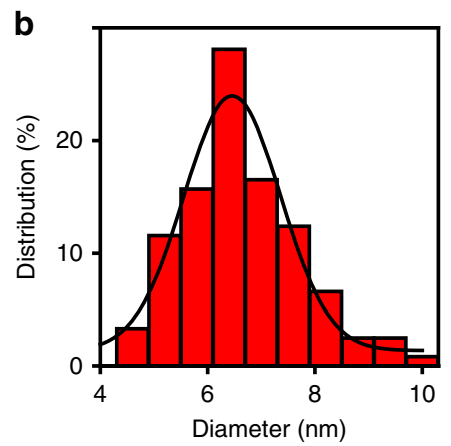

f

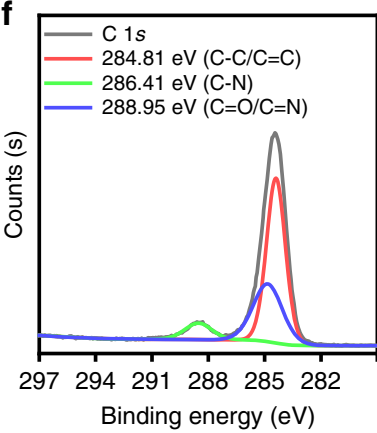

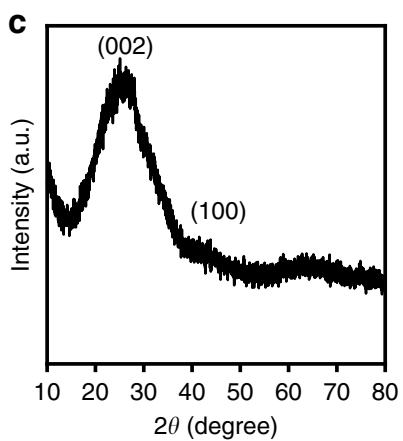

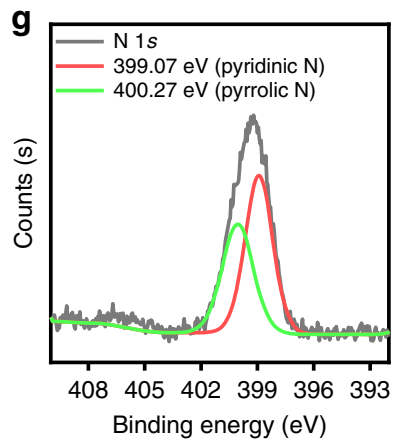



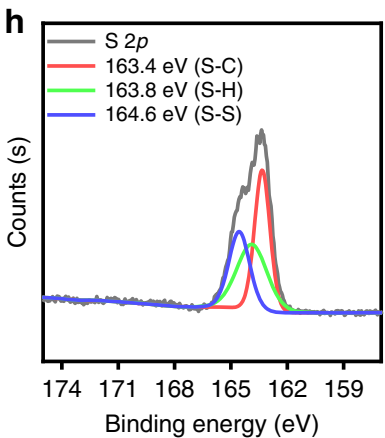


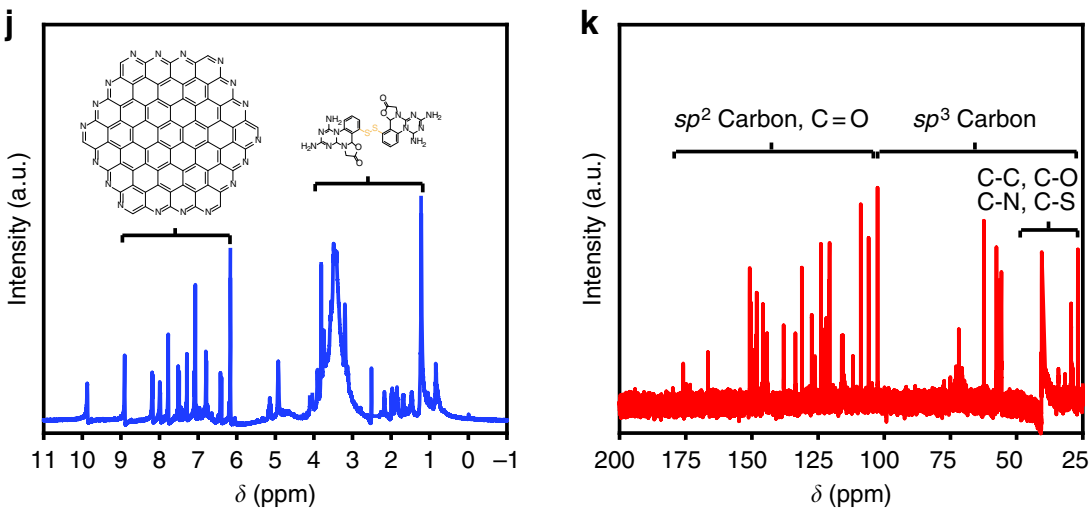

Fig. 2 Basic characterizations of H-CDs. a TEM image of the H-CDs, inset: high-resolution TEM (HR-TEM) image of the H-CDs. b Particles size distribution measured by TEM. $\mathbf{c}$ X-ray diffraction (XRD) pattern of the $\mathrm{H}$-CDs. d Raman spectrum of the $\mathrm{H}-\mathrm{CDs}$. e XPS spectrum and high-resolution $\mathbf{f} \mathrm{C} 1 \mathrm{~s}$, $\mathbf{g} \mathrm{N}$ 1s, and h S $2 p$ spectra of the H-CDs. i FT-IR spectrum of DTSA, MA and the H-CDs (the position marked by dotted rectangles refer to hydroxyl and amino group, peaks belong to disulfide bond are marked by dotted line). $\mathbf{j}^{1} \mathrm{H}$ NMR (insets: proposed structure of the $\mathrm{H}-\mathrm{CD}$ core and surface, the braces mark out the regions they belong to separately) and $\mathbf{k}{ }^{13} \mathrm{C}$ NMR spectra (the braces mark out the regions related to carbon different with different molecular orbital) of the H-CDs in DMSO-d6. Scale bars: $100 \mathrm{~nm}$ (a) and $10 \mathrm{~nm}$ (a-inset). H-CD, hydrophobic carbon dot, TEM transmission electron microscopy

due to their photon absorption and emission (Supplementary Fig. 2b, c) $)^{4,7,11,21,40,41}$. Visually, bright field and fluorescence microscopy images (Supplementary Fig. 3) of excess H-CD powder in ethanol indicate that thick stacking $\mathrm{H}-\mathrm{CD}$ powder produces red fluorescence, and the dissolved $\mathrm{H}-\mathrm{CD}$ solution displays blue emission. At the thin periphery of the H-CD powder infiltrated with the solution, the red and blue emission hybridize together, giving rise to a pink hybrid fluorescence $8,10,13$.

More intuitively, the photographs in Fig. 4a show that the pure as-prepared $\mathrm{H}-\mathrm{CD}$ solution and the solution with an addition of less than 50\% water (volume ratio) are orange to red homogeneous and transparent liquids. When the volume ratio of the water is greater than $50 \%$, the as-prepared $\mathrm{H}-\mathrm{CD}$ solution starts to separate the red powder out and turns into a turbid liquid with a suspension. Under a $365 \mathrm{~nm}$ UV excitation (Fig. 4b), the aforementioned transparent liquids display blue fluorescence, while the turbid liquid with the suspension glows red. In addition, correlated to the variation trend of the size distribution by adding more water, the intensity of the H-CD solution's blue emission decreases, while the red emission is heightened (Fig. 4c). The H$\mathrm{CDs}$ in solvents with different polarities exhibit a similar fluorescence phenomenon (Fig. 4d) to the as-prepared H-CDs with varying ratios of water. The red emission increases when the polarity of the solvent decreases. The UV-Vis absorption spectra and absorbance trend of the $\mathrm{H}-\mathrm{CD}$ solutions with varying ratios of water (Fig. 4e, f) reveal that with the injection of water, the absorbance at $360 \mathrm{~nm}$ continues decreasing, while a redshifted absorbance at $559 \mathrm{~nm}$ appears and continues increasing. This trend provides strong evidence for the presence of $\pi-\pi$ stacking in the H-CDs. Conjugated systems can form two distinct types of $\pi-\pi$ aggregates, a sandwich-type arrangement (H-aggregates) and a head-to-tail arrangement (J-aggregates) ${ }^{42}$. According to the molecular exciton coupling theory, the spectral redshift indicates that the H-CDs form J-aggregates, with a head-to-tail 

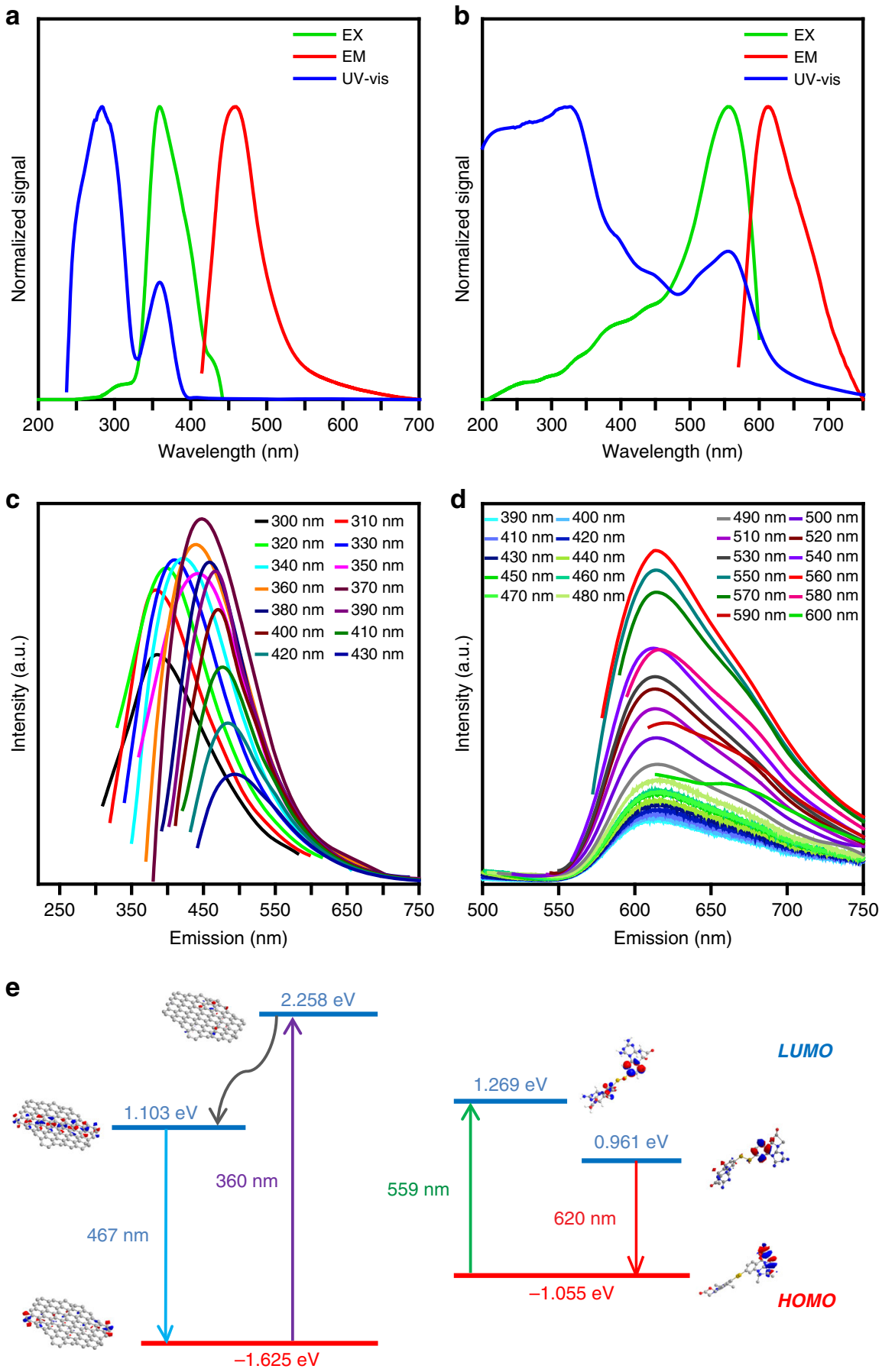

Fig. 3 Absorption and emission properties of $\mathrm{H}-\mathrm{CD}$ s and concerned orbital energy level. a UV - Vis absorption (blue line), PL excitation (EX) $\left(\lambda_{\mathrm{em}}=467\right.$ $\mathrm{nm}$, orange line), and emission (EM) $\left(\lambda_{\mathrm{ex}}=360 \mathrm{~nm}\right.$, red line) spectra of as-prepared H-CD solution. $\mathbf{b}$ UV-Vis absorption (green line), PL excitation (Ex) $\left(\lambda_{\mathrm{em}}=620 \mathrm{~nm}\right.$, orange line), and emission ( $\left.\mathrm{Em}\right)\left(\lambda_{\mathrm{ex}}=559 \mathrm{~nm}\right.$, red line) spectra of the H-CD powder. c, d PL emission spectra with different excitation wavelengths of $\mathrm{H}-\mathrm{CD}$ powder and as-prepared solution. e Energy level diagram of proposed H-CDs' graphitizing core, and the surface symmetrical heterocycle's molecular orbital. H-CD, hydrophobic carbon dot

arrangement ${ }^{43-46}$. Based on the red SSF of the H-CD powders, a fluorescent organic glass was fabricated and assembled into a WLED with a cyan LED-chip (Supplementary Fig. 4).

As mentioned above, when the $\mathrm{H}-\mathrm{CD}$ powders dissolve into DMF, an orange transparent liquid is obtained (Fig. $5 c$, left inset). Under $365 \mathrm{~nm}$ UV excitation, the H-CD powder DMF solution exhibits a pinkish red fluorescence (Fig. $5 \mathrm{c}$, right inset). The PL mapping spectrum (Fig. $5 \mathrm{c}$ ) reveals that there are both blue and red emission centers in the H-CD powder DMF solution.
Relatively, the H-CD powder acetic acid solution (Fig. 5a) has only a blue emission center, and the H-CD powder (Fig. $5 b$ ) solely obtains a red emission center. TEM images (Fig. 5d-f) indicate that the $\mathrm{H}-\mathrm{CD}$ aggregates, with an average diameter of approximately $56 \mathrm{~nm}$, exist in the DMF solution around the $\mathrm{H}$ $\mathrm{CD}$ monomers. The HR-TEM images (Fig. 5g-i) of the $\mathrm{H}-\mathrm{CD}$ aggregates, and the FFT diffraction pattern (inset of Fig. 5i) of the carbon lattice reveals there are different carbon lattice planes in the $\mathrm{H}-\mathrm{CD}$ aggregates, meaning that the $\mathrm{H}-\mathrm{CDs}$ assemble with 
a
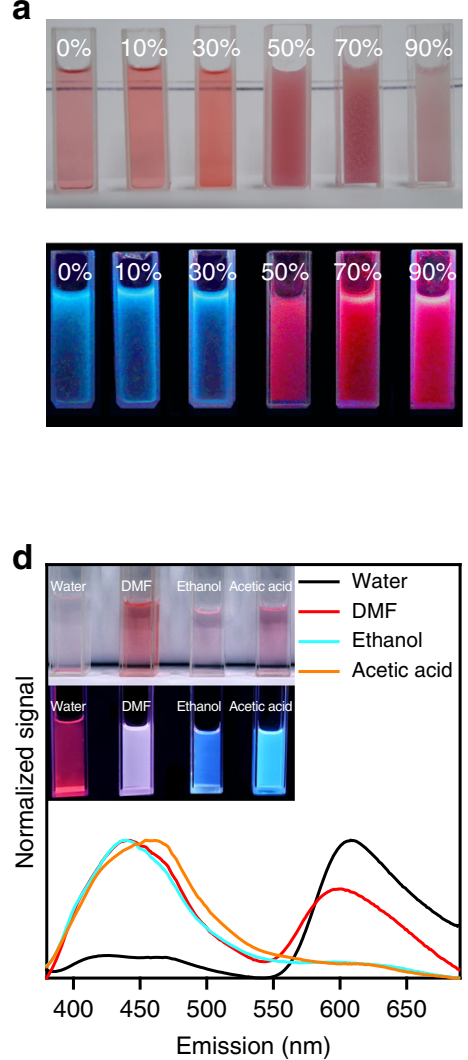
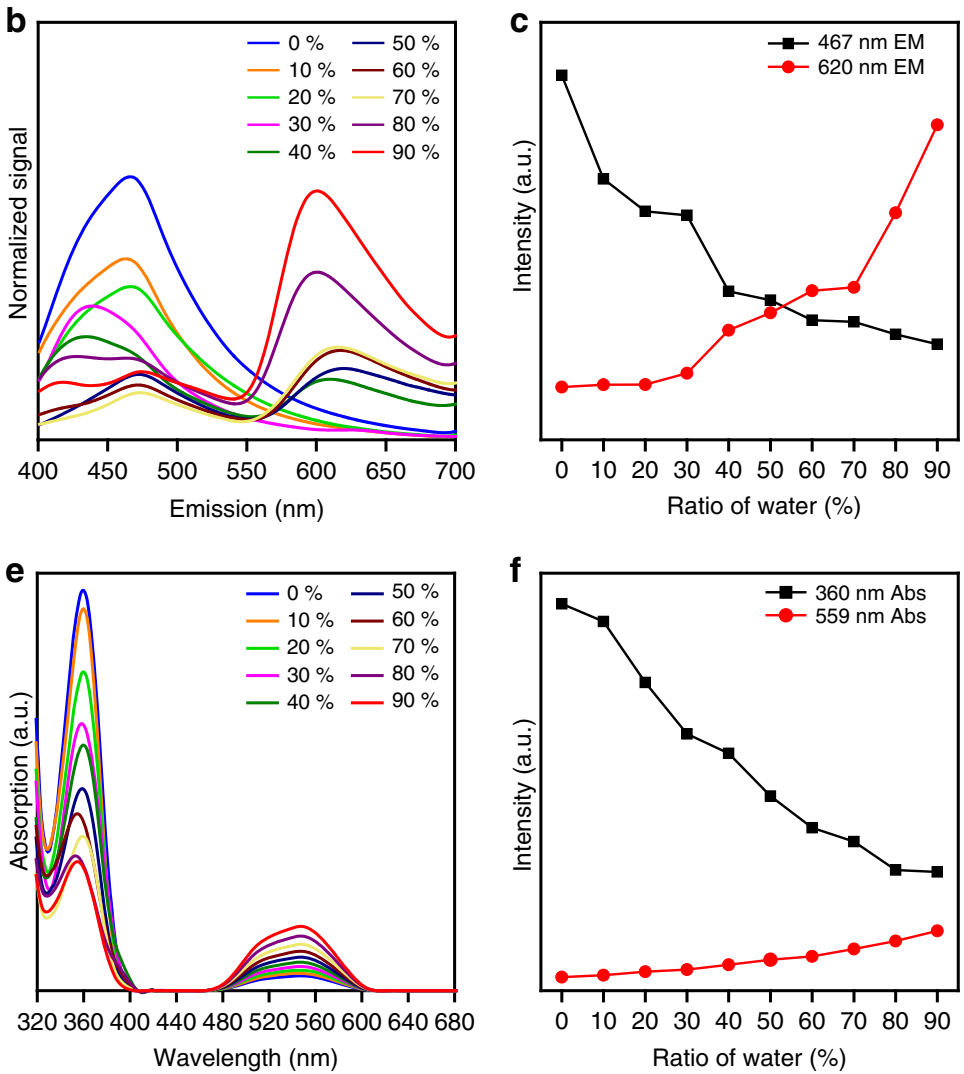

Fig. $4 \mathrm{H}$-CDs' absorption and emission varied with different solvent. a Photographs of the as-prepared $\mathrm{H}-\mathrm{CD}$ solution with varying volume ratios of water (from 0 to 90\%) under sunlight (top) and $365 \mathrm{~nm}$ ultraviolet radiation (bottom). b PL emission spectra of the H-CD as-prepared solution with varying ratios of water. c Trend of the H-CD as-prepared solutions' fluorescence intensity at 467 and 620 nm, varying with the ratio of water. $\mathbf{d}$ PL emission spectra of the H-CD powder solutions in different solvents (insets: photographs of the H-CD powder solutions in solvents with different polarities (from high to low) under sunlight (top) and $365 \mathrm{~nm}$ ultraviolet radiation (bottom)). e UV-Vis absorption spectra of the $\mathrm{H}$-CD as-prepared solution with different ratios of water. f Trend of the H-CD as-prepared solutions' absorbance at 360 and $559 \mathrm{~nm}$, varying with the ratio of water. $\mathrm{H}-\mathrm{CD}$, hydrophobic carbon dot

random orientation ${ }^{47-49}$. Therefore, the $\mathrm{H}-\mathrm{CD}$ aggregates generate red SSF and the monomers contribute to the blue emission, which reveals the relationship between the H-CDs' luminous mechanism and their dispersed state.

To further verify the structure and fluorescence mechanism of the $\mathrm{H}$-CDs, we carried out two control experiments, the first displaced DTSA with benzoate to remove the effect of the disulfide bonds. The produced CDs are named B-CDs. As shown in Supplementary Fig. 5a, c, the as-prepared B-CDs solution displays similar blue emission to the H-CDs. However, the B-CDs in the solid state exhibit no fluorescence (Supplementary Fig. 5b, d). Moreover, the solid-state B-CDs can easily dissolve into water (Supplementary Fig. 5e). According to the FT-IR spectra of BCDs and H-CDs, the chemical structure of the B-CDs is similar to the H-CDs, except for the disulfide bonds. Thus, the relationship between the symmetrical surface around the disulfide bonds and the H-CDs' hydrophobicity and red AIE can be affirmed.

The second control experiment used a postmodification method to synthesize dithiosalicylic acid-modified CDs, which are named P-CDs. First, MA is dissolved into acetic acid and undergoes a solvothermal pretreatment. The P-CD intermediate is water-soluble and displays blue fluorescence (Supplementary Fig. 6a). The TEM image of this intermediate in Supplementary Fig. $6 \mathrm{~d}$ and its inset indicates that a carbonized dot structure with a $0.25 \mathrm{~nm}$ lattice spacing (111 lattice plane of carbon), which can further verify that the blue emission of the $\mathrm{H}$-CDs comes from its carbonized core. P-CDs were then fabricated by mixing DTSA with the aforementioned intermediate and acetic acid, after a post-solvothermal processing. As shown in Supplementary Fig. 6b, c, e, f, P-CDs exhibit same hydrophobicity and PL properties as the $\mathrm{H}-\mathrm{CD}$, which confirms the root of the H-CDs' hydrophobicity and red AIE is the DTSA-modified surface.

Therefore, we can build a model comprised of the core formed by $\mathrm{MA}$ with an $\mathrm{N}, \mathrm{S}, \mathrm{O}$-containing, rotatable symmetrical heterocyclic surface. Optical properties and calculated energy level transitions reveal the correspondence of blue emission to the core and red emission to the surface. Photoluminescence videos (Supplementary Movies 1, 2, 3) of the H-CDs in different dispersed-states suggest that $\mathrm{H}$-CDs show blue emission in a dissolved state, and red emission in a solid state. The $\mathrm{H}-\mathrm{CD}$ ethanol solution was added onto a copper grid and dried, then deionized water was sprayed onto the copper grid. The TEM image (Supplementary Fig. 7) of the copper grid revealed that the $\mathrm{H}-\mathrm{CD}$ monomers were becoming closer than $\mathrm{H}-\mathrm{CD}$ solution displayed in Fig. 2a. Thus, a convincible aggregate and luminous mechanism can be proposed: in solution, H-CDs' graphitized cores are dominant while the rotatable symmetrical heterocycles around the disulfide bond is recessive; therefore, the H-CD solution exhibits excitation-correlated blue fluorescence, similar to reported carbon dots. When the $\mathrm{H}-\mathrm{CD}$ monomers contact water, the hydrophobicity of their surfaces cause them to approach each other. Then the conjugated system of the surfaces conducts $\pi-\pi$ stacking to overlay each other. Finally, the H-CDs take the shape of J-aggregates. Due to this aggregation, the graphitized cores will suffer a $\pi-\pi$ stacking interaction and further turn off the blue emission via ACQ. Furthermore, the 

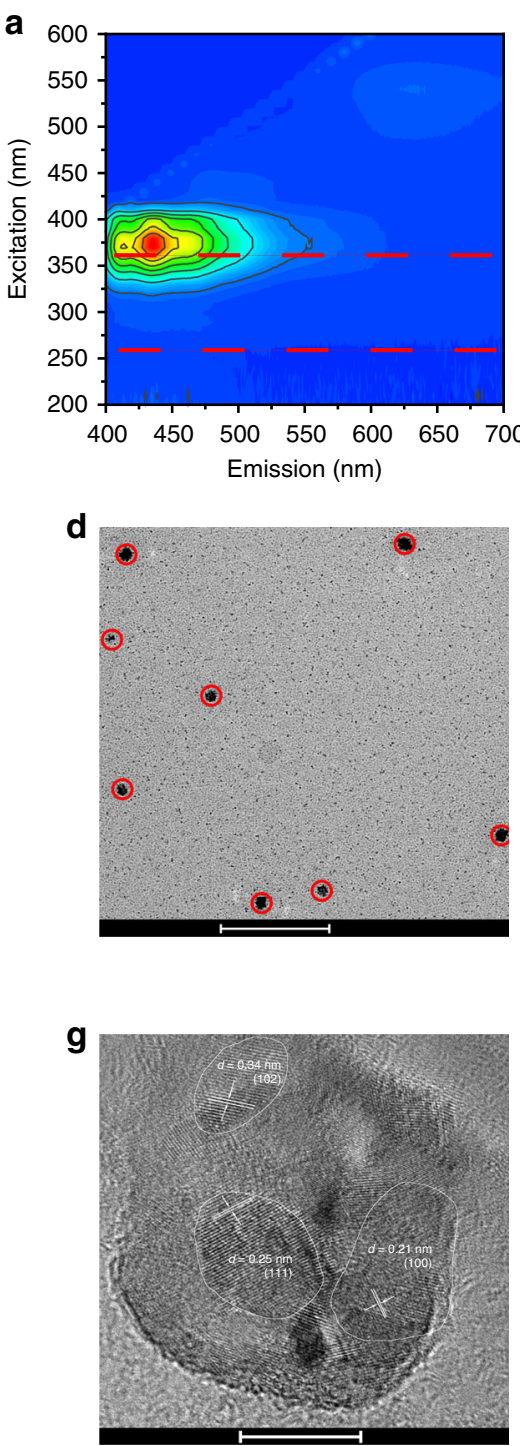
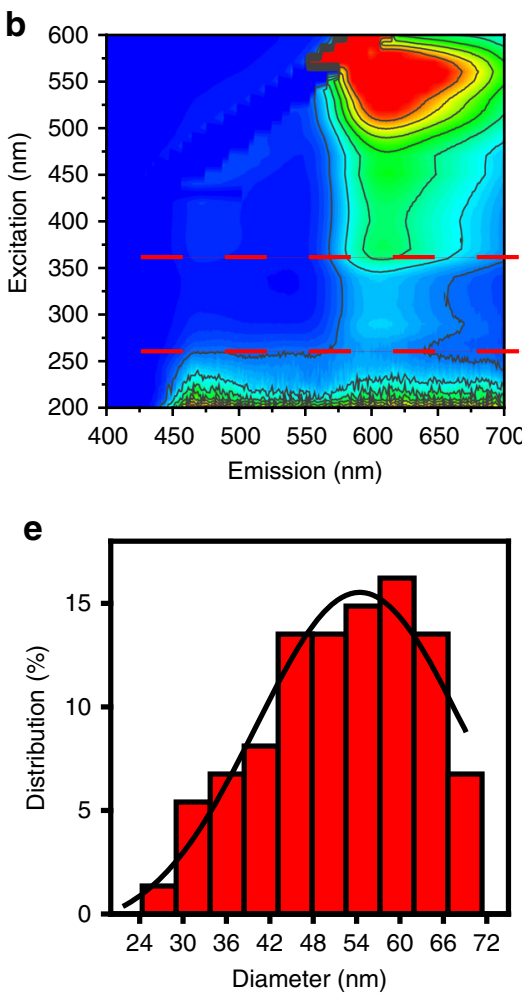

h

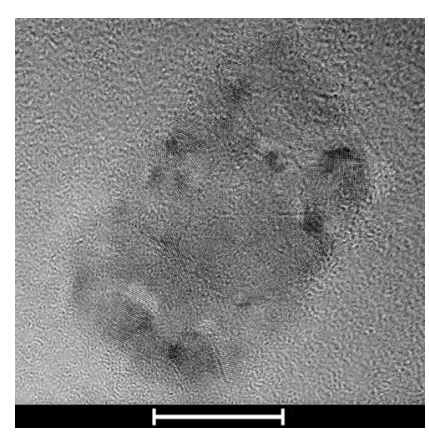

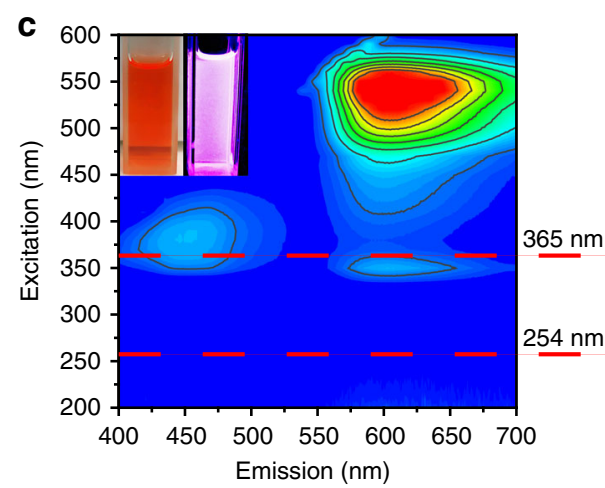

$\mathbf{f}$

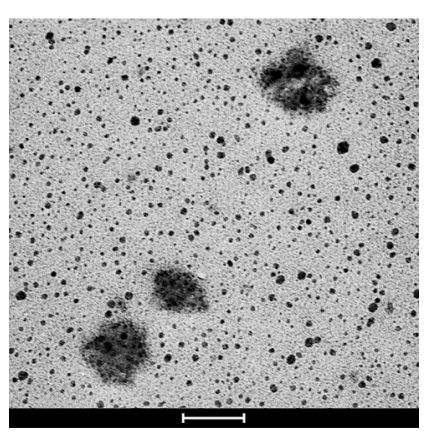

i

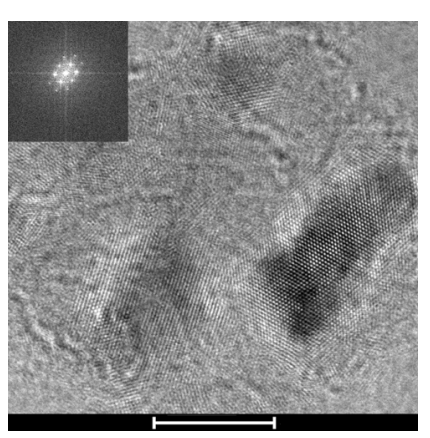

Fig. 5 Different fluorescence and morphology of $\mathrm{H}-\mathrm{CD}$ in varying state. PL mapping spectra of a the H-CD powder acetic acid solution, $\mathbf{b} \mathrm{H}-\mathrm{CD}$ powder and $\mathbf{c}$ the H-CD powder DMF solution (insets: photos of the H-CD DMF solution under white light (left) and $365 \mathrm{~nm}$ (right) irradiation, dash line marks out emission under 365 or $254 \mathrm{~nm}$ UV). d, f TEM image and e particles size distribution of H-CD aggregates (marked by red circles) in the DMF solution. g-i High-resolution TEM (HR-TEM) image of the H-CD aggregates in the DMF solution, inset: FFT diffraction pattern of carbon lattice. Scale bars: 500 nm (d), $50 \mathrm{~nm}(\mathbf{f}), 20 \mathrm{~nm}(\mathbf{h})$ and $10 \mathrm{~nm}(\mathbf{g}, \mathbf{i}) . \mathrm{H}-\mathrm{CD}$, hydrophobic carbon dot, TEM transmission emission microscopy

axisymmetric heterocycles shown in Supplementary Fig. 2 suffer from the restriction of intramolecular rotation (RIR) of symmetrical heterocycles about their disulfide bonds axes, like other reported symmetrical molecules with $\mathrm{AIE}^{17,18,50}$, resulting in red $\mathrm{AIE}^{15,16,51}$

H-CD-based two-switch-mode luminescence ink. As shown in Fig. 6a, the as-prepared $\mathrm{H}-\mathrm{CD}$ solution was painted on a filter paper. Under white light, it is almost colorless and shows a blue fluorescence under $365 \mathrm{~nm}$ UV excitation. At $254 \mathrm{~nm}$ the UV irradiation cannot produce any fluorescence, which conforms to the PL property of the H-CD monomers shown in Fig. 5. By adding water and air-drying, its fluorescence under $365 \mathrm{~nm} \mathrm{UV}$ turns to pink. Furthermore, it appears as a red fluorescence, which suggests the $\mathrm{H}$-CDs on the filter paper contains both $\mathrm{H}-\mathrm{CD}$ monomers and $\mathrm{H}-\mathrm{CD}$ aggregates, compared with the former data. With the addition of ethanol and air-drying, the liquid $\mathrm{H}-\mathrm{CD}$ displays the same optical properties as the $\mathrm{H}-\mathrm{CD}$ monomers. Furthermore, the addition of water can turn on the red emission again. This phenomenon suggests that the asprepared H-CD solution can be utilized as a reversible twoswitch-mode ink. A schematic mechanism for the ink is shown in Fig. $6 \mathrm{~b}$. The square frames in Fig. $6 \mathrm{~b}$ represent the filter paper, the wavy lines represent the paper's fibers. Blue dots represent the $\mathrm{H}$ $\mathrm{CD}$ monomers dispersed in the filter paper due to the restriction of the paper's fibers. As mentioned above, H-CD monomers cannot be excited at $254 \mathrm{~nm}$ but can be excited at $365 \mathrm{~nm}$. When water is introduced, some of the $\mathrm{H}-\mathrm{CD}$ monomers aggregate and surface. Furthermore, the other monomers remain joint to the fibers. Therefore, under $365 \mathrm{~nm}$ irradiation, both the monomers in the filter paper and the aggregates on surface can be excited to glow blue and red emission, which display as a hybrid pink fluorescence. While under $254 \mathrm{~nm}$ irradiation, the monomers are not excited further, resulting in the red emission only. Once ethanol is applied, the aggregates will dissolve into the filter paper as monomers again; therefore, this process is reversible. A video has been taken to show this reversible process (Supplementary 



Fig. 6 Presentation and principle of H-CDs' reversible dual-fluorescence. a Photographs of filter papers with different treatment under variable irradiation. b Schematic diagram of the $\mathrm{H}-\mathrm{CD}$ s precipitated with water addition and redissolving into ethanol, with the resulting multimode fluorescence. (The square frames represent the filter paper, the wavy lines represent the paper's fibers, the blue dots represent the H-CD monomers, the flash symbols upward refer to admit light while the downwards ones present emission, the red crosses mean no emission). $\mathrm{H}-\mathrm{CD}$, hydrophobic carbon dot

Movie 4). In this video, we observed that the transfer of the different fluorescence is extremely fast. The excellent reversibility of the process makes the H-CDs promising candidates for practical anticounterfeiting and encryption applications.

Finally, the as-prepared H-CD solution was filled into an empty mark pen to form a convenient anticounterfeiting and encryption tool. Two school badges painted with a commercially available highlighter pen (CAHP) and an $\mathrm{H}-\mathrm{CD}$ as-prepared solution-filled mark pen (HMP) (Fig. 7a) based on the filter papers were fabricated. The badges underwent the same treatments as Fig. 6a, c in order. Under white light, the badges are as white as empty filter papers. The CAHP-painted badge exhibits cyan fluorescence under $365 \mathrm{~nm}$ UV and blue emission under $254 \mathrm{~nm}$ UV. Additionally, water addition does not make an obvious change. While under the different treatments and irradiation, the HMP-painted badge can display four different luminescence characteristics (with HMP, under $365 \mathrm{~nm} \mathrm{UV}$, blue emission; under $254 \mathrm{~nm}$ UV, no emission; with HMP and

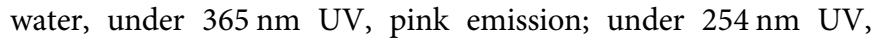
red emission). Evidently, the H-CD as-prepared solution-filled mark pen manifests distinctly unique luminescent properties and stability through the injection of different solvents. The HMP dual-encryption utilization is presented in Fig. 7b. "SC", "US" and "NU" are painted by HMP; moreover, "C", "S" and 
a
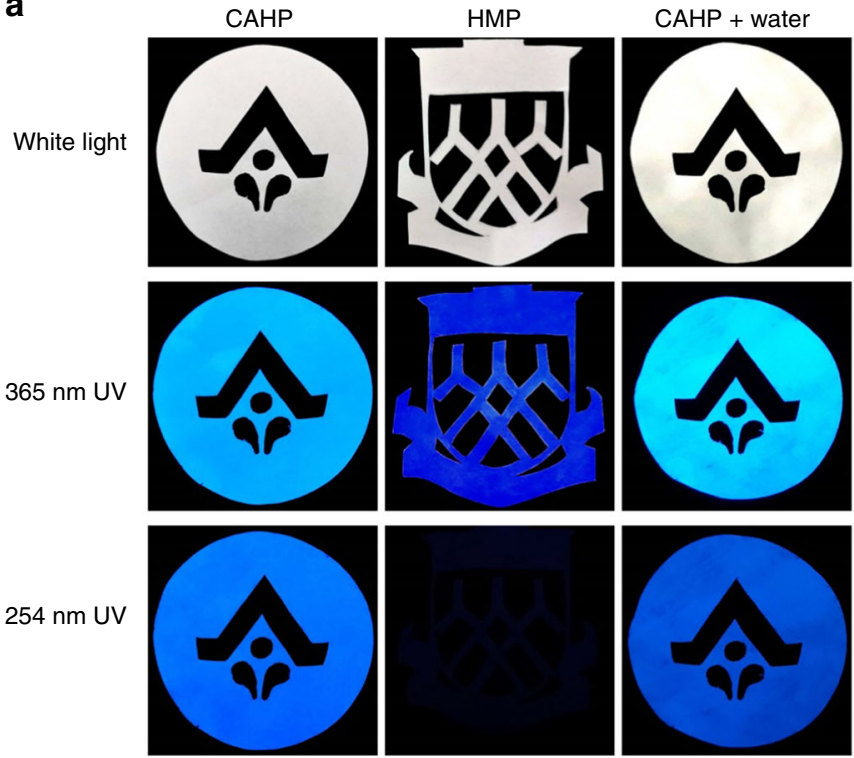

b White light

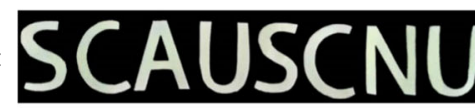

$365 \mathrm{~nm}$ UV

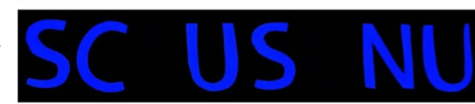

$254 \mathrm{~nm}$ UV
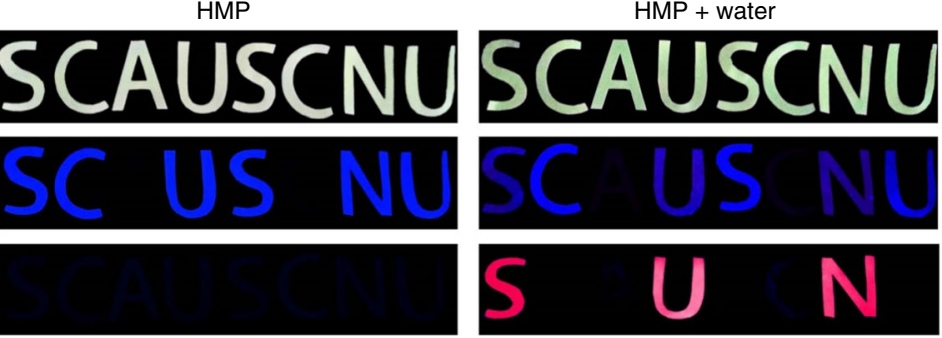

Fig. 7 Application of $\mathrm{H}-\mathrm{CDs}$ ink. a H-CD as-prepared solution-filled mark pen (HMP) utilized as an anticounterfeiting badge compared with commercially available highlighter pen (CAHP); b HMP utilized as a dual-encryption badge. $\mathrm{H}-\mathrm{CD}$, hydrophobic carbon dot

" $U$ " are covered with wax after the ink is air-dried. With $365 \mathrm{~nm}$ UV excitation, with or without water, only a series of meaningless fake-codes are shown in blue fluorescence. With 254 nm UV irradiation and no water addition, only darkness is observed. Specifically, the true code "SUN" appears as red fluorescence with simultaneous water treatment and $254 \mathrm{~nm}$ UV excitation.

\section{Discussion}

In summary, $\mathrm{H}$-CDs with reversible two-switch-mode luminescence (blue dissolved fluorescence and red AIE) are attained from an eco-friendly, low-cost one-pot solvothermal treatment. TEM, XRD and Raman spectra were taken to confirm the nature of the H-CD carbon nanoparticles. Afterwards, the hydrophobic disulfide bond, containing a symmetrical heterocyclic surface of H-CDs was confirmed by XPS, FT-IR spectra and NMR. The hydrophobicity stems from the abundance of pyridinic and epoxy groups on their symmetrical heterocyclic surfaces. As dispersed monomers in organic solvents, such as AC and ethanol, the asprepared CDs display a "traditional" ultraviolet absorption $\left(\lambda_{\mathrm{EX}}=315 \mathrm{~nm}\right)$ and blue emission $\left(\lambda_{\mathrm{EM}}=467 \mathrm{~nm}\right)$. The asprepared CDs are extremely hydrophobic because of the epoxy and pyridyl groups on their surfaces. Thus, the as-prepared CDs will precipitate if water is injected. The precipitations show no blue emission, as the CDs aggregate into J-aggregates and the carbonized cores suffer from aggregation-caused-quenching (ACQ) due to the $\pi-\pi$ stacking interaction of their vast conjugated system. Furthermore, the dominated surficial energy transition turns into the production of fluorescence, as the intramolecular rotation of the symmetrical heterocycles about the disulfide bonds is restricted, leading to red AIE $\left(\lambda_{\mathrm{EM}}=621 \mathrm{~nm}\right.$, $\left.\lambda_{\mathrm{EX}}=559 \mathrm{~nm}\right)$. This mechanism is confirmed by the aforementioned characterization methods as well as experiments on monomers and aggregates of $\mathrm{H}-\mathrm{CD}$ in a DMF solution: pure $\mathrm{H}$ $\mathrm{CD}$ monomers in $\mathrm{AC}$ or ethanol solutions solely exhibit blue emission; pure H-CD powder only show red AIE; in a DMF solution, when the monomers and aggregates of H-CD coexist, the blue dissolved fluorescence and red AIE take place. Two control experiments were conducted to further confirm this theory. The turning off of the H-CDs' blue emission and turning on of the red AIE is reversible. As a result, an anticounterfeiting fluorescence ink for advanced anticounterfeiting and dual encryption has been fabricated based on the two-switch-mode luminescence of $\mathrm{H}-\mathrm{CD}$.

\section{Methods}

Materials. Melamine, dithiosalicylic acid, and benzoate were obtained from Shanghai Adamas Reagent Co., Ltd. Acetic acid was purchased from Guangdong Guanghua Sci-Tech Co., Ltd. All reagents were of analytical grade and used directly without further purification. Deionized water was produced through a Millipore water purification system (Milli-Q, Millipore) and used throughout the study.

Instruments and measurements. UV-Vis absorption spectra of the powder samples were performed using a Shimadzu UV-2550 ultraviolet-visible spectrophotometer. PL spectra were measured using a Hitachi FL7000 fluorescence spectrophotometer instrument apparatus. The XRD pattern was collected using a XD-2×/M4600. The HR-TEM images were recorded using a JEOL-2010 electron microscope. FT-IR spectra were taken on a Nicolet Avatar 360 FT-IR spectrophotometer. X-ray photoelectron spectroscopy (XPS) experiments were performed using a Kratos AXIS Ultra DLD X-ray photoelectron spectrometer with a monochromatic $\mathrm{Al} \mathrm{Ka} \mathrm{X}$-ray source. Raman spectra were obtained by a Renishaw via a 
microspectrometer with an excitation wavelength of $785 \mathrm{~nm}$ laser. Particle size analysis is achieved from a Malvern Nano 2SE laser particle size analyzer. NMR measurements were taken by AVANCEIII500 (Bruker). The absolute quantum yield and lifetime are respectively measured by a Hamamatsu C11347 and a Quantaurus Tau C11367.

Synthesis of the H-CDs. $201.6 \mathrm{mg}$ MA and $544 \mathrm{mg}$ DTSA were dissolved into 40 $\mathrm{mL}$ acetic acid with ultrasonic treatment, then the solution was transferred into an $80 \mathrm{~mL}$ Teflon reactor and kept at $180^{\circ} \mathrm{C}$ for $10 \mathrm{~h}$ in an air oven. After the solvothermal treatment, the as-prepared $\mathrm{H}-\mathrm{CD}$ solution was added into $1 \mathrm{~L}$ boiled water to form $\mathrm{H}-\mathrm{CD}$ powder and wash out the residual raw materials and solvent. Finally, purified H-CD powder was achieved through vacuum filtration. To confirm the reliability of this water-wash method, we applied column chromatography to purify the H-CD solution for comparison, and the H-CDs obtained from this approach are named CC-H-CDs. FT-IR and UV-Vis spectra of H-CDs and CC$\mathrm{H}-\mathrm{CD}$ powders in Supplementary Fig. 8 suggest the components are approximately identical. Therefore, the water-wash method is considered as reliable as column chromatography.

Synthesis of the P-CDs. $201.6 \mathrm{mg}$ MA was dissolved into $40 \mathrm{~mL}$ acetic acid with ultrasonic treatment, then the solution was transferred into an $80 \mathrm{~mL}$ Teflon reactor and maintained at $180^{\circ} \mathrm{C}$ for $5 \mathrm{~h}$ in an air oven. The as-prepared solution was purified by centrifuge and column chromatography and intermediate powders were collected from freeze-drying. Afterwards, $150 \mathrm{mg}$ intermediate powder and $544 \mathrm{mg}$ DTSA were dissolved into $40 \mathrm{~mL}$ acetic acid, transferred into an $80 \mathrm{~mL}$ Teflon reactor, and maintained at $180^{\circ} \mathrm{C}$ for $5 \mathrm{~h}$ in an air oven. After the solvothermal treatment, the as-prepared $\mathrm{H}-\mathrm{CD}$ solution was added into $1 \mathrm{~L}$ boiled water to form H-CD powders and wash out residual raw materials. Finally, the purified P-CDs powder was obtained through vacuum filtration.

Synthesis of the B-CDs. $201.6 \mathrm{mg}$ MA and $434 \mathrm{mg}$ were dissolved into $40 \mathrm{~mL}$ acetic acid with ultrasonic treatment, then transferred into an $80 \mathrm{~mL}$ Teflon reactor and maintained at $180^{\circ} \mathrm{C}$ for $10 \mathrm{~h}$ in an air oven. After the solvothermal treatment, the as-prepared B-CDs solution was dialyzed in deionized water for a week to remove residual raw materials. Finally, the purified solid-state B-CDs were obtained through freeze-drying.

Preparation of H-CD-powders-based fluorescence organic glass. $150 \mathrm{mg} \mathrm{H}-\mathrm{CD}$ powders, $53.7 \mathrm{mg}$ dibenzoyl peroxide, $1 \mathrm{~mL}$ dibutyl phthalate and $15 \mathrm{~mL}$ methyl methacrylate were added into a $250 \mathrm{~mL}$ flask, the mixture was maintained at 90 $-92^{\circ} \mathrm{C}$ for $15 \mathrm{~min}$ in water bath. Afterwards, the flask was cooled down to $40^{\circ} \mathrm{C}$ rapidly, and the mixture was poured into a template and maintained at $100{ }^{\circ} \mathrm{C}$ for $2 \mathrm{~h}$. After reverse molding, fluorescent organic glasses (Supplementary Fig. 4) based on $\mathrm{H}-\mathrm{CD}$ powders were obtained.

\section{Computational process of H-CDs' molecular orbital energy level and fluor-} escence lifetime. The energy level transitions (Fig. 3e) of the H-CDs' carbonized cores and symmetrical heterocyclic surfaces were calculated by the formula

$$
E=h \frac{c}{\lambda}
$$

according to their absorptive and emissive properties, which fit the molecular orbital energy level of the proposed structure simulated by the Gaussian 09 plug-in in ChemBioOffice $2014^{\circledast}$. The fluorescence decay curve and double-exponential fitting results are shown in Supplementary Fig. 2a. The fitting function is shown in the formula below

$$
y=y_{0}+A_{1} * \exp \left(-\left(x-x_{0}\right) / t_{1}\right)+A_{2} \times \exp \left(-\left(x-x_{0}\right) / t_{2}\right)
$$

and the fluorescence lifetime is calculated as $4.56 \mathrm{~ns}$ by the formula below:

$$
t=\left(A_{1} t_{1}^{2}+A_{2} t_{2}^{2}\right) /\left(A_{1} t_{1}+A_{2} t_{2}\right)=4.56 \mathrm{~ns} .
$$

\section{Data availability}

Data are available from the corresponding author upon reasonable request.

Received: 15 September 2018 Accepted: 25 March 2019

Published online: 17 April 2019

\section{References}

1. Wang, Z. et al. 53\% efficient red emissive carbon quantum dots for high color rendering and stable warm white-light-emitting diodes. Adv. Mater. 29, 1702910 (2017).
2. Wang, B. et al. Self-assembled core-shell-corona multifunctional non-viral vector with AIE property for efficient hepatocyte-targeting gene delivery. Polym. Chem. 8, 7486-7498 (2017).

3. Jiang, K. et al. Red, green, and blue luminescence by carbon dots: full-color emission tuning and multicolor cellular imaging. Angew. Chem. Int. Ed. 54, 5360-5363 (2015).

4. Yuan, F. et al. Multicolor fluorescent graphene quantum dots colorimetrically responsive to all-pH and a wide temperature range. Nanoscale 7, 11727-11733 (2015).

5. Song, Y. et al. Drug-derived bright and color-tunable N-doped carbon dots for cell imaging and sensitive detection of $\mathrm{Fe}^{3+}$ in living cells. ACS Appl. Mater. Interfaces 9, 7399-7405 (2017).

6. Miao, X. et al. Synthesis of carbon dots with multiple color emission by controlled graphitization and surface functionalization. Adv. Mater. 30, 1704740 (2018).

7. Hu, S., Trinchi, A., Atkin, P. \& Cole, I. Tunable photoluminescence across the entire visible spectrum from carbon dots excited by white light. Angew. Chem. Int. Ed. 54, 2970-2974 (2015).

8. He, J. et al. Solid-state carbon dots with red fluorescence and efficient construction of dual-fluorescence morphologies. Small 13, 1700075 (2017).

9. Ge, J. et al. Red-emissive carbon dots for fluorescent, photoacoustic, and thermal theranostics in living mice. Adv. Mater. 27, 4169-4177 (2015).

10. Zhang, Y. et al. A self-quenching-resistant carbon nanodot powder with multicolored solid-state fluorescence for ultra-fast staining of various representative bacterial species within one minute. Nanoscale 8, 19744-19753 (2016).

11. Shao, J. et al. Full-color emission polymer carbon dots with quench-resistant solid-state fluorescence. Adv. Sci. 4, 1700395 (2017).

12. Lou, Q. et al. Water-triggered luminescent "nano-bombs" based on supra(carbon nanodots). Adv. Mater. 27, 1389-1394 (2015).

13. Chen, Y. et al. A self-quenching-resistant carbon-dot powder with tunable solid-state fluorescence and construction of dual-fluorescence morphologies for white light-emission. Adv. Mater. 28, 312-318 (2016).

14. Spano, F. C. The spectral signatures of Frenkel polarons in H- and Jaggregates. Acc. Chem. Res. 43, 429-439 (2010).

15. Yeh, H. C., Wu, W. C. \& Chen, C. T. The colourful fluorescence from readilysynthesised 3,4-diaryl-substituted maleimide fluorophores. Chem. Commun. 3, 404-405 (2003)

16. Wang, Y. et al. Aggregation-induced emission luminogen with deep-red emission for through-skull three-photon fluorescence imaging of mouse. ACS Nano 11, 10452-10461 (2017)

17. Tang, B. Z. et al. Efficient blue emission from siloles. J. Alloy. Compd. 11, 2974-2978 (2001).

18. Hong, Y. \& Lam, J. W. \& Tang, B. Z. Aggregation-induced emission: phenomenon, mechanism and applications. Chem. Commun. 29, 4332-4353 (2009). https://doi.org/10.1039/B904665H

19. Zhu, S. et al. Highly photoluminescent carbon dots for multicolor patterning, sensors, and bioimaging. Angew. Chem. Int. Ed. 52, 3953-3957 (2013).

20. Wang, T. Y., Chen, C. Y., Wang, C. M., Tan, Y. Z. \& Liao, W. S. Multicolor functional carbon dots via one-Step refluxing synthesis. ACS Sens. 2, 354-363 (2017).

21. Lu, S. et al. Piezochromic carbon dots with two-photon fluorescence. Angew Chem. Int. Ed. 56, 6187-6191 (2017).

22. Lai, L. F. et al. One-step synthesis of NH2-graphene from in situ grapheneoxide reduction and its improved electrochemical properties. Carbon 49 , 3250-3257 (2011)

23. Tan, L. et al. Synthesis of highly bright oil-soluble carbon quantum dots by hot-injection method with $\mathrm{N}$ and B Co-Doping. J. Nanosci. Nanotechnol. 16, 2652-2657 (2016)

24. Tan, J., Zhang, J., Li, W., Zhang, L. Q. \& Yue, D. M. Synthesis of amphiphilic carbon quantum dots with phosphorescence properties and their multifunctional applications. J. Mater. Chem. C 4, 10146-10153 (2016).

25. Panniello, A. et al. Luminescent oil-soluble carbon dots toward white light emission: a spectroscopic study. J. Phys. Chem. C 122, 839-849 (2017).

26. Nandi, S. et al. Membrane analysis with amphiphilic carbon dots. Chem. Commun. 50, 10299-10302 (2014).

27. Moon, B. J. et al. Facile and purification-free synthesis of nitrogenated amphiphilic graphitic carbon dots. Chem. Mater. 28, 1481-1488 (2016)

28. Lu, L. et al. Hydrophobic-carbon-dot-based dual-emission micelle for ratiometric fluorescence biosensing and imaging of $\mathrm{Cu}^{2+}$ in liver cells. Biosens. Bioelectron. 92, 101-108 (2017).

29. Kozak, O. et al. Surfactant-derived amphiphilic carbon dots with tunable photoluminescence. J. Phys. Chem. C 117, 24991-24996 (2013).

30. Kong, D. et al. Amphiphilic carbon dots for sensitive detection, intracellular imaging of $\mathrm{Al}^{3+}$. Anal. Chim. Acta 953, 63-70 (2017).

31. Jones, S. S., Sahatiya, P. \& Badhulika, S. One step, high yield synthesis of amphiphilic carbon quantum dots derived from chia seeds: a solvatochromic study. New J. Chem. 41, 13130-13139 (2017). 
32. $\mathrm{Gu}, \mathrm{J}$. J. et al. Green synthesis of amphiphilic carbon dots from organic solvents: application in fluorescent polymer composites and bio-imaging. RSC Adv. 8, 12556-12561 (2018).

33. Fowley, C. et al. Highly luminescent biocompatible carbon quantum dots by encapsulation with an amphiphilic polymer. Chem. Commun. 48, 9361-9363 (2012).

34. Chen, J. et al. Understanding the capsanthin tails in regulating the hydrophilic-lipophilic balance of carbon dots for a rapid crossing cell membrane. Langmuir 33, 10259-10270 (2017).

35. Li, F. et al. Selenium-doped carbon quantum dots for free-radical scavenging. Angew. Chem. Int. Ed. 56, 9910-9914 (2017).

36. Geng, S. et al. Synthesis of lipid-black phosphorus quantum dot bilayer vesicles for near-infrared-controlled drug release. Chem. Commun. 54, 6060-6063 (2018).

37. Fan, $X$. et al. Deoxygenation of exfoliated graphite oxide under alkaline conditions: a green route to graphene preparation. Adv. Mater. 20, 4490-4493 (2008).

38. Ding, D. et al. Ultrabright organic dots with aggregation-induced emission characteristics for real-time two-photon intravital vasculature imaging. $A d v$. Mater. 25, 6083-6088 (2013).

39. Zheng, M. et al. One-pot to synthesize multifunctional carbon dots for nea infrared fluorescence imaging and photothermal cancer therapy. ACS Appl. Mater. Interfaces 8, 23533-23541 (2016).

40. Yeh, T. F. et al. Elucidating quantum confinement in graphene oxide dots based on excitation-wavelength-independent photoluminescence. J. Phys. Chem. Lett. 7, 2087-2092 (2016).

41. Cai, S. et al. Visible-light-excited ultralong organic phosphorescence by manipulating intermolecular interactions. Adv. Mater. 29, 1701244 (2017)

42. Deng, Y. H., Feng, X. J., Yang, D. J., Yi, C. H. \& Qiu, X. Q. $\pi-\pi$ stacking of the aromatic groups in lignosulfonates. Bioresources 7, 1145-1156 (2012).

43. Deng, Y. et al. Investigation of aggregation and assembly of alkali lignin using iodine as a probe. Biomacromolecules 12, 1116-1125 (2011).

44. Li, X. et al. Self-assembled graphene quantum dots induced by cytochrome c: a novel biosensor for trypsin with remarkable fluorescence enhancement. Nanoscale 5, 7776-7779 (2013).

45. Ma, Z. et al. Seeking brightness from nature: J-aggregation-induced emission in cellulolytic enzyme lignin nanoparticles. ACS Sustain. Chem. Eng. 6, 3169-3175 (2018).

46. Su, M. et al. Nano-assemblies from J-aggregated dyes: a stimuliresponsive tool applicable to living systems. J. Am. Chem. Soc. 141, 402-413 (2019).

47. Xie, Z., Du, Q., Wu, Y., Hao, X. \& Liu, C. Full-band UV shielding and highly daylight luminescent silane-functionalized graphene quantum dot nanofluids and their arbitrary polymerized hybrid gel glasses. J. Mater. Chem. C 4, 9879-9886 (2016).

48. Zhang, Z. et al. New transparent flexible nanopaper as ultraviolet filter based on red emissive Eu(III) nanofibrillated cellulose. Opt. Mater. 73, 747-753 (2017)

49. Feng, X. et al. Use of carbon dots to enhance UV-blocking of transparent nanocellulose films. Carbohydr. Polym. 161, 253-260 (2017).

50. Li, Y. et al. Aggregation induced red shift emission of phosphorus doped carbon dots. RSC Adv. 7, 32225-32228 (2017).
51. Chen, C. et al. Highly luminescent Inks: aggregation-induced emission of copper-iodine hybrid clusters. Angew. Chem. Int. Ed. 57, 7106-7110 (2018).

\section{Acknowledgements}

The present work was supported by the National Natural Science Foundation of China (Grant Nos. 21571067, 51402207, 61675072, 11874021), the National Natural Science Foundation of China Joint Fund with Guangdong under Key Project (Grant No. U1501242) and the Teamwork Projects funded by the Guangdong Natural Science Foundation (Grant No. S2013030012842)

\section{Author contributions}

H.Y., C.H., Y.L. and Z.L. conceived the experiments. H.Y. synthesized all materials, made all measurements and wrote the paper. B.L., J.Z. and X.Z. gave suggestions to optimize the system. All authors discussed the results and commented on the manuscript at all stages.

\section{Additional information}

Supplementary Information accompanies this paper at https://doi.org/10.1038/s41467019-09830-6.

Competing interests: The authors declare no competing interests.

Reprints and permission information is available online at http://npg.nature.com/ reprintsandpermissions/

Journal peer review information: Nature Communications thanks Zaicheng Sun, and the other anonymous reviewer(s) for their contribution to the peer review of this work. Peer reviewer reports are available.

Publisher's note: Springer Nature remains neutral with regard to jurisdictional claims in published maps and institutional affiliations.

(c) (i) Open Access This article is licensed under a Creative Commons BY Attribution 4.0 International License, which permits use, sharing adaptation, distribution and reproduction in any medium or format, as long as you give appropriate credit to the original author(s) and the source, provide a link to the Creative Commons license, and indicate if changes were made. The images or other third party material in this article are included in the article's Creative Commons license, unless indicated otherwise in a credit line to the material. If material is not included in the article's Creative Commons license and your intended use is not permitted by statutory regulation or exceeds the permitted use, you will need to obtain permission directly from the copyright holder. To view a copy of this license, visit http://creativecommons.org/ licenses/by/4.0/.

(C) The Author(s) 2019 